\title{
Current research on ADS at the Joint Institute for Nuclear Research
}

\author{
J. Adam ${ }^{a, b}$, K. Katovsky ${ }^{c}$, R. Vespalec ${ }^{a, d}$, L. Zavorka ${ }^{a}$, M. Zeman ${ }^{a, c *}$ \\ a Joint Institute for Nuclear Research, Joliot-Curie 6, 141980 Dubna, Russian Federation \\ ${ }^{b}$ Nuclear Physics Institute of the ASCR, v. v. i., Rez 130, 25068 Rez, Czech Republic \\ ${ }^{c}$ Brno University of Technology, Technicka 3058/10, 61600 Brno, Czech Republic \\ ${ }^{d}$ Czech Technical University in Prague, v Holesovickach 2, 18000 Prague, Czech Republic \\ E-mail: iadamejinr.ru, katovsky@feec.vutbr.cz, vespalec@jinr.ru, \\ zavorka@jinr.ru, zemandjinr.ru
}

\section{A. Baldin ${ }^{a}, e$, W. Furman ${ }^{a}$, J. Khushvaktov ${ }^{a}$, A. Solnyshkin ${ }^{a}$, J. Svoboda $^{a, c}$, P. Tichy ${ }^{a, b, d}$ V. Tsoupko-Sitnikov ${ }^{a}$, S. Tyutyunikov ${ }^{a}$, J. Vrzalovaa ${ }^{a, b}$, V. Wagner ${ }^{b, d}$, P. Zhivkov ${ }^{f}$ \\ e Institute for Advanced Studies "OMEGA", Universitetskaya 19, 141980 Dubna, Moscow region, Russian Federation \\ ${ }^{f}$ Institute of Nuclear Research and Nuclear Energy of Bulgarian Academy of Sciences, Tzarigradsko chaussee, Blvd. 72, 1784 Sofia, Bulgaria}

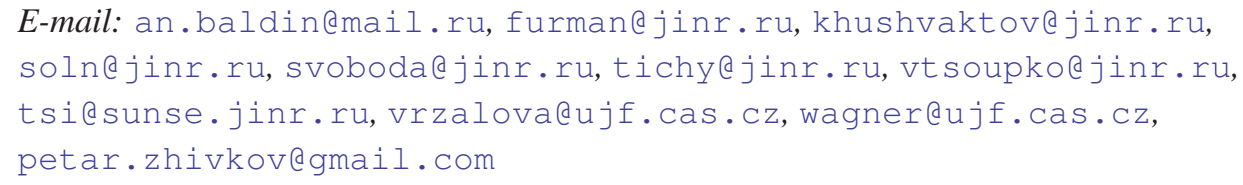

The research on Accelerator Driven Systems (ADS) has more than 20 year's tradition at the Joint Institute for Nuclear Research. Since 2010, the most experiments have been performed with a spallation target composed of $512 \mathrm{~kg}$ of natural uranium. This target called QUINTA was irradiated with proton and deuteron beams of high energies. Currently, final preparations of a new spallation target BURAN consisting of 21 tons of depleted uranium are under way. The main tasks of the project are experimental investigation of neutron production inside the spallation target, possibility of natural thorium utilization and transmutation of the minor actinides and long-lived fission products. The supplementary field of interest is a measurement of nuclear data and verification of nuclear codes and theoretical models related to the ADS technologies.

4th Workshop on ADS and thorium

31 August - 2 September 2016

University of Huddersfield, England

* Speaker. This work was financially supported by Ministry of Youth and Education and Ministry of Finances of the Czech Republic. The project was also supported from the LO1210, FEKT-S-14-2520 projects at the BUT. The author is the Brno Ph.D. Talent Scholarship Holder - Funded by the Brno City Municipality. 


\section{Introduction and motivation}

One of the most important problems of current nuclear power plants is spent nuclear fuel (SNF), which is produced in a volume of thousands tons per year world wide. A possible solution to decrease volume of spent nuclear fuel could be in Accelerator Driven Systems $(A D S)$ and transmutation technologies. Each ADS consists of a particle accelerator and sub-critical spallation target.

The investigation of the transmutation of SNF has started at the Joint Institute for Nuclear Research $(J I N R)$ at the beginning of $90^{\circ}$. The research of the ADS at the JINR is conducted within the collaboration Energy and Transmutation of Radioactive Waste (E\&TRAW). The group is interested in experimental research on the transmutation of SNF in the spallation target. The second topic is validation of nuclear data important for ADS, mainly determination of the crosssection for different materials, which are irradiated by various particles with wide energy spread and determination of the neutron flux inside spallation targets. All experiments are simulated using different nuclear models with the help Monte Carlo simulations.

The experiments are focused on the Relativistic Nuclear Technology $(R N T)$. The scheme of RNT is very similar to the ADS one, but there are a few differences. The main ones are the increase of the energy of the primary beam up to $10 \mathrm{GeV}$ and the material of the spallation target is the same as the blanket material.

One of the mains goals of experimental program is an estimation of parameters of secondary neutron flux inside the spallation targets.

\section{Experimental facility}

The part of the E\&T RAW group which belongs to Dzhelepov's Laboratory of Nuclear Problems (DLNP) works with two particle accelerators and six spallation targets placed at the JINR.

\subsection{Accelerator fleet}

The first experiments were performed with particle accelerator called Nuclotron, which is located at the Veksler and Baldin Laboratory of High Energy Physics (VBLHEP). The accelerator is superconductive synchrotron and it was built between 1987-1992. The accelerating ring has diameter $215.5 \mathrm{~m}$ and the mass is about 80 tons. The accelerator can accelerate protons up to energy $12.8 \mathrm{GeV}$ and heavy ions up to maximal energy $5 \mathrm{AGeV}$. The intensity of the beam is $10^{11}$ per pulse and the input power is $1.5 \mathrm{MW}$. The experiments with all used spallation targets were held at the Nuclotron accelerator.

Experimental accelerator Phasotron is placed in DLNP at the JINR. Phasotron was reconstructed from first accelerator synchrocyclotron which was built in 1949. The reconstruction took placed between 1979-1984 and at the end the accelerator can produce protons accelerated on energy $660 \mathrm{MeV}$ with current $1.6 \mu \mathrm{A}$. Phasotron produce about $10^{13}$ protons per one second which is its advance. The accelerator is mainly used for a medical therapy. Last two years the group of E\&T RAW prepared a set of experiments with experimental set-up QUINTA. 


\subsection{Spallation targets}

The group involved in the ADS experiments in JINR performed these experiments using six spallation targets. The first experiment with a spallation target was in 1993, where the group irradiated target called GAMMA-2, was composed in order to investigate the neutron production in a spallation target. GAMMA-2 contained a lead target of $200 \mathrm{~mm}$ in length and $80 \mathrm{~mm}$ in diameter. The target was surrounded by paraffin of thickness $60 \mathrm{~mm}$. Basic information and results are showed in $[1,2]$. The second spallation target was called GAMMA-3. The target was made of lead surrounded with 25 graphite blocks. The parameters of the set-up were $1100 \times 1100$ x 600 $\mathrm{mm}^{3}$. The GAMMA-3 was irradiated with relativistic beams at Nuclotron accelerator [3]. Since 2005, the experiments were performed with set-up Energy and Transmutation. Experiments with the spallation target were performed for research of fast spallation and fission neutrons. The set-up was composed of lead target and uranium blanket. The target contained four identical sections with hexagonal shape. Energy and Transmutation contained lead target and uranium blanket. The weight of metallic uranium is $204 \mathrm{~kg}$. The set-up was irradiated by proton and deuteron beams produced by Nuclotron accelerator. Few experiments are described in [4, 5]. Recent experiments have been performed with QUINTA made of natural uranium.

\section{Spallation target QUINTA}

The spallation set-up QUINTA contains five hexagonal sections with natural uranium in metallic form. The first section consists of 54 aluminium rods $36 \mathrm{~mm}$ in diameter and $104 \mathrm{~mm}$ in length. In the center of the target there is a $80 \mathrm{~mm}$ wide beam window. Uranium rods are fixed in $5 \mathrm{~mm}$ thick aluminium plates. Other 4 sections are similar and contain 61 cylinders of natural uranium. The total mass of metallic natural uranium is $512 \mathrm{~kg}$. Each two sections are divided by $17 \mathrm{~mm}$ air gap which are used for placement of the experimental samples. The total length of the QUINTA is $638 \mathrm{~mm}$ and the high is $350 \mathrm{~mm}$. The scheme of the set-up is shown in the figure 1. The set-up is surrounded by $100 \mathrm{~mm}$ thick lead bricks shielding. The spallation target QUINTA was irradiated at the Nuclotron accelerator by proton, deuteron and carbon particle by different energies and at the Phasotron accelerator, which accelerate protons at energy $660 \mathrm{MeV}$. Several experiments performed at the QUINTA target are described in following publications $[6,7,8,9]$.

\section{Target Buran}

Future plans of the group are to perform experiments with spallation target Buran. The word Buran is an abbreviation of russian words Bolshoi Uran (great uranium). The spallation target is made of metallic depleted uranium, which is arranged in cylindrical shape in steel container. The total weight of the uranium is 21 tons. The diameter of the target is $1200 \mathrm{~mm}$ and the length is $1000 \mathrm{~mm}$. Buran target has 72 experimental canals, which can be used for placement of the samples. Other information connected to the Buran target including can be found in [9]. The advantages of the target are a simple geometry and expected minimal leakage of the secondary neutrons. The first experiment with target Buran is planned at the beginning of 2017 at the Phasotron accelerator. 

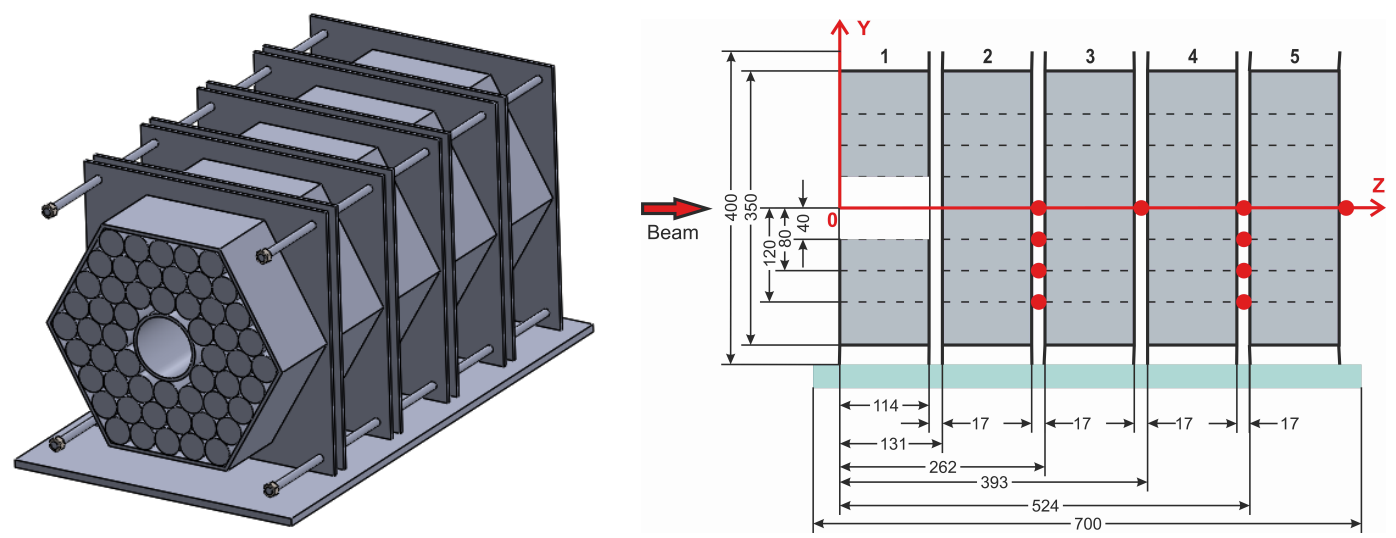

Figure 1: The main view of the QUINTA target and side view with experimental samples of ${ }^{59} \mathrm{Co}$ and dimensions

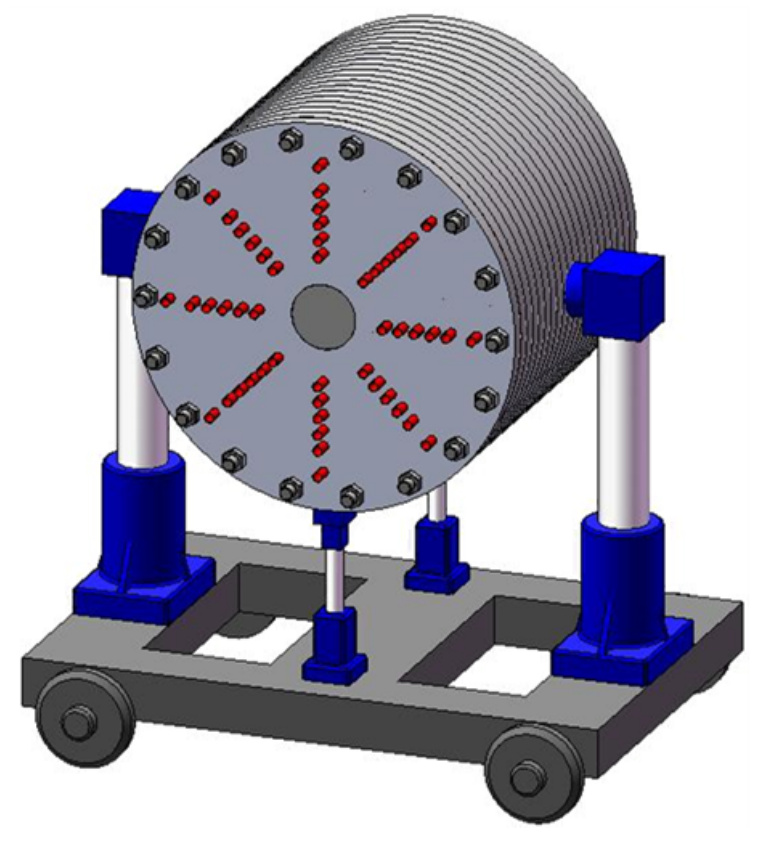

Figure 2: Spallation target Buran[9]

\subsection{Spectroscopic equipment}

The irradiated samples are transported in a lead container to the spectroscopic complex YaSNaPP2 and measured using high purity germanium detectors. The group in DLNP has 6 detectors, one of them is a planar detector and five of them are coaxial detectors (both $\mathrm{P}$ and $\mathrm{N}$ types) made by ORTEC and CANNBERA companies with relative efficiencies up to $35 \%$. The detectors are calibrated before each experiment with using standard gamma-ray sources and the calibrated energy interval is from $5 \mathrm{keV}$ up to $3 \mathrm{MeV}$. Each irradiated sample is measured at least 6 times for better analysis and the time of measurement starts from few minutes on the beginning up to several days. The spectra are measured with maximal statistical uncertainty $1 \%$ for followed peaks and the dead time is kept under $10 \%$. Usually, the measurements of irradiated samples started approximately 
10 minutes (in the case of Phasotorn acelerator) or 60 minutes (in the case of Nuclotron acelerator) after the end of irradiation.

\section{Data analysis}

The measured data are analysed using spectroscopic software Deimos32[10] able to determine gamma lines energies and their areas. The output of the program is used for determination of experimental reaction rates from products of reactions in irradiated samples. The equation for determination of experimental reaction rate with correction factors, which is shown in 3.1. We used in-house developed software package (unpublished) for determination of experimental reaction rates $R_{\text {exp }}$.

$$
R_{\text {exp }}=\frac{S\left(E_{\gamma}\right) \cdot C_{a b s}\left(E_{\gamma}\right) \cdot B_{a}}{I_{\gamma} \cdot \varepsilon_{p}\left(E_{\gamma}\right)} \frac{t_{\text {real }}}{t_{\text {live }}} \frac{1}{N_{A}} \frac{1}{N_{P}} \frac{\mathrm{e}^{\lambda \cdot t_{0}}}{1-\mathrm{e}^{-\lambda \cdot t_{\text {real }}}} \frac{\lambda \cdot t_{\text {irr }}}{1-\mathrm{e}^{-\lambda \cdot t_{\text {irr }}}} \frac{1}{C_{\text {coisum }}},
$$

where $S\left(E_{\gamma}\right)$ - the peak area, $C_{a b s}$ - the self-absorption correction, $B_{a}$-the beam intensity correction, $I_{\gamma}$ - the $\gamma$-line intensity per decay, $\varepsilon_{p}\left(E_{\gamma}\right)$ - the detector efficiency, $t_{\text {real }}$ - the real measurement time, $t_{\text {live }}$-the time of measurement, $N_{A}$-the number of atoms in the sample, $N_{P}$ - the number of particles during irradiation, $t_{0}$ - the time between end of irradiation and start of measurement, $\lambda$ - decay constant, $t_{i r r}$ - the time of irradiation and $C_{\text {coisum }}$ - is the correction for coincidence summing.

\subsection{Determination of the neutron flux}

The experimental determination of the mean value of the neutron flux is obtained with help of the reaction rate of threshold reactions and it comes from basic equation by reaction rate- $R$ which is shown in equation 3.2. The equation 3.2 represents integral of the reaction rate, where the energetically dependent values are at the right side. The dependent values are the cross-section $\sigma\left(E_{n}\right)$ and the neutron flux $\varphi\left(E_{n}\right)$.

$$
R=\int_{E_{t h}}^{\infty} \sigma\left(E_{n}\right) \cdot \varphi\left(E_{n}\right) \mathrm{d} E_{n}
$$

The equation means that the mean value of the neutron flux in the energy interval between the threshold $(n, x n)$ reaction and the maximum neutron energy constant can be calculated as a ratio of experimental reaction rate and cross section. After calculation of the neutron flux in the above mentioned interval the neutron flux between the efficiency threshold energy of $(n,(x-1) n)$ and $(n, x n)$ reaction can be calculated using the neutron flux from the last energy interval. The experimental part is focused on determination of the neutron flux with using ${ }^{59} \mathrm{Co}$. The detailed steps of analysis are explained in a paper [11].

\section{Example of experimental part of research}

Part of the experimental work is focused on determination of the neutron flux in the spallation target QUINTA with the use of threshold activation detectors. The spallation target QUINTA was irradiated at the VBLHEP by the deuterons with energy $8 \mathrm{GeV}$ which were produced by the 
Nuclotron accelerator. The spallation target was irradiated for 27 hours and 18 minutes and the number of particles was $6.11(8) \cdot 10^{12}$. The positions of the 10 samples of ${ }^{59} \mathrm{Co}$ which were irradiated in the secondary neutron field are shown at the figure 1 . The positions of samples were behind section 2, 3,4 and 5 in the beam center of the QUINTA target and in position $-40,-80$ and $-120 \mathrm{~mm}$ behind sections 2 and 4 .

The products are ordered from the residual nuclei with the lowest threshold energy, in this case ${ }^{59} \mathrm{Co}(n, \gamma){ }^{60} \mathrm{Co}$, up to the reaction with the highest threshold energy, for the product ${ }^{59} \mathrm{Co}(n, 4 n){ }^{56} \mathrm{Co}$. The table 1 shows the threshold energies and other information. The results of the residual nuclei from ${ }^{59} \mathrm{Co}$ are shown in table 2, for experimental samples placed at different sections in the beam center, and in table 3 for experimental samples which were situated at different positions behind sections number 2 and 4 . The highest reaction rates were measured behind section two at position $40 \mathrm{~mm}$ from the beam center.

\begin{tabular}{|c|c|c|c|c|}
\hline $\begin{array}{c}\text { Products } \\
\text { nuclei }\end{array}$ & $\begin{array}{c}\boldsymbol{E}_{\text {th }} \\
(\mathbf{M e V})\end{array}$ & $\begin{array}{c}\boldsymbol{E}_{\text {thEff }} \\
(\mathbf{M e V})\end{array}$ & $\boldsymbol{T}_{\mathbf{1} / \mathbf{2}}$ & $\begin{array}{c}\varphi_{\mathbf{i}} \\
(\mathbf{M e V})\end{array}$ \\
\hline${ }^{59} \mathrm{Co}(n, \gamma){ }^{60} \mathrm{Co}$ & 0.00 & 0.14 & $5.27 \mathrm{y}$ & $0.14-3.9$ \\
\hline${ }^{59} \mathrm{Co}(n, p){ }^{59} \mathrm{Fe}$ & 0.8 & 3.9 & $44.45 \mathrm{~d}$ & $3.9-10.4$ \\
\hline${ }^{59} \mathrm{Co}(n, 2 n){ }^{58} \mathrm{Co}$ & 10.6 & 10.4 & $70.9 \mathrm{~d}$ & $10.4-16.5$ \\
\hline${ }^{59} \mathrm{Co}(n, 3 n){ }^{57} \mathrm{Co}$ & 19.4 & 16.5 & $271.8 \mathrm{~d}$ & $16.5-31.9$ \\
\hline${ }^{59} \mathrm{Co}(n, 4 n){ }^{56} \mathrm{Co}$ & 30.9 & 31.9 & $77.3 \mathrm{~d}$ & 31.9 \\
\hline
\end{tabular}

Table 1: Products of ${ }^{59} \mathrm{Co}$ with table and efficiency threshold energy and half-life

\begin{tabular}{|c|c|c|c|c|}
\hline \multirow{2}{*}{$\begin{array}{c}\text { Products } \\
\text { of reaction }\end{array}$} & \multicolumn{4}{|c|}{$\boldsymbol{Z}(\mathbf{m m}), \mathbf{Y}=\mathbf{0 ~} \mathbf{~ m m}$} \\
\cline { 2 - 5 } & $\mathbf{2 5 4}$ & $\mathbf{3 8 5}$ & $\mathbf{5 1 6}$ & $\mathbf{6 4 7}$ \\
\hline${ }^{60} \mathrm{Co}$ & $13.6(14)$ & $10.3(11)$ & $7.27(78)$ & $3.82(42)$ \\
\hline${ }^{59} \mathrm{Fe}$ & $2.19(23)$ & $1.33(14)$ & $0.754(80)$ & $0.336(37)$ \\
\hline${ }^{58} \mathrm{Co}$ & $18.7(20)$ & $11.1(12)$ & $6.50(69)$ & $2.90(31)$ \\
\hline${ }^{57} \mathrm{Co}$ & $5.60(56)$ & $3.50(37)$ & $2.00(21)$ & $1.05(11)$ \\
\hline${ }^{56} \mathrm{Co}$ & $1.03(11)$ & $0.604(64)$ & $0.356(38)$ & $0.142(18)$ \\
\hline
\end{tabular}

Table 2: Experimental reaction rates $\left(\right.$ atom $^{-1} \cdot$ deuteron $\left.^{-1} \cdot 10^{-27}\right)$ at the center of the QUINTA target

\begin{tabular}{|c|c|c|c|}
\hline \multirow{2}{*}{$\begin{array}{c}\text { Products } \\
\text { of reaction }\end{array}$} & \multicolumn{3}{|c|}{$Y(\mathbf{m m}), \mathbf{Z}=\mathbf{2 6 2} \mathbf{~ m m}$} \\
\cline { 2 - 4 } & $\mathbf{- 4 0}$ & $\mathbf{- 8 0}$ & $\mathbf{- 1 2 0}$ \\
\hline${ }^{60} \mathrm{Co}$ & $14.9(16)$ & $10.9(11)$ & $9.31(98)$ \\
\hline${ }^{59} \mathrm{Fe}$ & $1.60(17)$ & $0.715(77)$ & $0.378(42)$ \\
\hline${ }^{58} \mathrm{Co}$ & $1.21(13)$ & $0.410(37)$ & $0.203(23)$ \\
\hline${ }^{57} \mathrm{Co}$ & $3.47(38)$ & $1.39(16)$ & $0.719(77)$ \\
\hline${ }^{56} \mathrm{Co}$ & $0.580(65)$ & $0.164(19)$ & $0.88(13)$ \\
\hline
\end{tabular}

\begin{tabular}{|c|c|c|c|}
\hline \multirow{2}{*}{$\begin{array}{c}\text { Products } \\
\text { of reaction }\end{array}$} & \multicolumn{3}{|c|}{$Y(\mathbf{m m}), \mathbf{Z}=\mathbf{5 1 6} \mathbf{~ m m}$} \\
\cline { 2 - 4 } & $\mathbf{- 4 0}$ & $\mathbf{- 8 0}$ & $\mathbf{- 1 2 0}$ \\
\hline${ }^{60} \mathrm{Co}$ & $7.27(76)$ & $6.00(67)$ & $5.20(55)$ \\
\hline${ }^{59} \mathrm{Fe}$ & $0.649(69)$ & $0.348(39)$ & $0.225(42)$ \\
\hline${ }^{58} \mathrm{Co}$ & $5.03(53)$ & $2.56(37)$ & $1.58(34)$ \\
\hline${ }^{57} \mathrm{Co}$ & $1.53(18)$ & $0.77(10)$ & $0.512(89)$ \\
\hline${ }^{56} \mathrm{Co}$ & $0.231(26)$ & $0.115(16)$ & $0.070(12)$ \\
\hline
\end{tabular}

Table 3: Experimental reaction rates $\left(\right.$ atom $^{-1} \cdot$ deuteron $\left.^{-1} \cdot 10^{-27}\right)$ at the different sections 2 and 4 and different positions of the QUINTA target

The experimental neutron flux was calculated from reaction with the highest threshold energy, reaction ${ }^{59} \mathrm{Co}(n, 4 n){ }^{56} \mathrm{Co}$, up to reaction rate with the lowest threshold energy, reaction ${ }^{59} \mathrm{Co}(n, \gamma){ }^{60} \mathrm{Co}$. Experimentally determined values were compared with simulations performed in MCNPX2.7 transport code [12] using the Liege intra-nuclear cascade model (INCL4.2) [13, 14] 
merged with a standard version KHSv3p of ABLA evaporation/fission model developed in GSI Darmstadt [15].

The results are shown in table 4, table 5 and figure 3 . The highest experimental neutron flux was calculated at the position $-40 \mathrm{~mm}$ from the beam center behind section number 2 . The neutron flux was increasing with decreasing vertical and horizontal distance from the beam center. The agreement between experimental and calculated values for reaction ${ }^{59} \mathrm{Co}(n, \gamma){ }^{60} \mathrm{Co},{ }^{59} \mathrm{Co}(n, 2 n){ }^{58} \mathrm{Co}$ and ${ }^{59} \mathrm{Co}(n, 3 n){ }^{57} \mathrm{Co}$ is in range to five standard deviations. The ratio of ${ }^{59} \mathrm{Co}(n, p){ }^{59} \mathrm{Fe}$ and ${ }^{59} \mathrm{Co}(n, 4 n){ }^{56} \mathrm{Co}$ reactions are not in a good agreement.

\begin{tabular}{|c|c|c|c|c|}
\hline \multirow{2}{*}{ Fluxes } & \multicolumn{4}{|c|}{$\boldsymbol{Z}(\mathbf{m m}), \boldsymbol{Y}=\mathbf{0 ~} \mathbf{m m}$} \\
\cline { 2 - 5 } & $\mathbf{2 6 2}$ & $\mathbf{3 9 3}$ & $\mathbf{5 2 4}$ & $\mathbf{6 3 8}$ \\
\hline$\varphi_{1 \text { Exp }}$ & $790(83)$ & $597(63)$ & $423(45)$ & $222(25)$ \\
$\varphi_{1 \text { Cal }} / \varphi_{1 \text { Exp }}$ & $0.698(73)$ & $0.852(90)$ & $0.894(95)$ & $1.09(12)$ \\
\hline$\varphi_{2 \operatorname{Exp}}$ & $6.35(67)$ & $3.96(42)$ & $2.18(23)$ & $1.03(11)$ \\
$\varphi_{2 \text { Cal }} / \varphi_{2 \operatorname{Exp}}$ & $7.41(80)$ & $8.72(93)$ & $10.5(11)$ & $7.92(90)$ \\
\hline$\varphi_{3 \operatorname{Exp}}$ & $1.78(19)$ & $0.96(10)$ & $0.592(62)$ & $0.162(20)$ \\
$\varphi_{3 \text { Cal }} / \varphi_{3 \operatorname{Exp}}$ & $1.45(15)$ & $1.81(19)$ & $1.84(20)$ & $1.42(17)$ \\
\hline$\varphi_{4 \operatorname{Exp}}$ & $0.695(75)$ & $0.50(5)$ & $0.268(28)$ & $0.220(20)$ \\
$\varphi_{4 \text { Cal }} / \varphi_{4 \operatorname{Exp}}$ & $1.10(15)$ & $1.31(14)$ & $1.52(16)$ & $0.410(37)$ \\
\hline$\varphi_{5 \operatorname{Exp}}$ & $0.224(23)$ & $0.132(14)$ & $0.0788(83)$ & $0.0310(38)$ \\
$\varphi_{5 \text { Cal }} / \varphi_{5 \operatorname{Exp}}$ & $0.459(48)$ & $0.459(48)$ & $0.525(56)$ & $0.310(37)$ \\
\hline
\end{tabular}

Table 4: Experimental values of the neutron flux in different positions (neutron $\cdot$ deuteron ${ }^{-1} \cdot \mathrm{MeV}^{-1} \cdot \mathrm{cm}^{-2}$. $\left.10^{-3}\right)$ and comparison with simulation at the center $(\mathrm{Y}=0 \mathrm{~mm})$ of QUINTA target

\begin{tabular}{|c|c|c|c|}
\hline \multirow{2}{*}{ Fluxes } & \multicolumn{3}{|c|}{$\boldsymbol{Y}(\mathbf{m m}), \mathbf{Z}=\mathbf{2 6 2} \mathbf{m m}$} \\
\cline { 2 - 4 } & $\mathbf{- 4 0}$ & $\mathbf{- 8 0}$ & $\mathbf{- 1 2 0}$ \\
\hline$\varphi_{1 \text { Exp }}$ & $868(95)$ & $636(69)$ & $544(57)$ \\
$\varphi_{1 \text { Cal }} / \varphi_{1 \text { Exp }}$ & $0.638(70)$ & $0.671(72)$ & $0.579(61)$ \\
\hline$\varphi_{2 \operatorname{Exp}}$ & $4.81(52)$ & $2.30(15)$ & $1.31(14)$ \\
$\varphi_{2 \text { Cal }} / \varphi_{2 \operatorname{Exp}}$ & $8.10(87)$ & $9.6(11)$ & $9.2(10)$ \\
\hline$\varphi_{3 \operatorname{Exp}}$ & $1.21(13)$ & $0.410(37)$ & $0.203(23)$ \\
$\varphi_{3 \text { Cal }} / \varphi_{3 \operatorname{Exp}}$ & $0.810(86)$ & $1.92(20)$ & $1.74(20)$ \\
\hline$\varphi_{4 E x p}$ & $0.533(55)$ & $0.338(37)$ & $0.168(15)$ \\
$\varphi_{4 \text { Cal }} / \varphi_{4 \operatorname{Exp}}$ & $0.910(10)$ & $0.88(10)$ & $0.80(10)$ \\
\hline$\varphi_{5 \operatorname{Exp}}$ & $0.126(14)$ & $0.0357(42)$ & $0.0192(24)$ \\
$\varphi_{5 \text { Cal }} / \varphi_{5 \operatorname{Exp}}$ & $0.601(67)$ & $0.838(99)$ & $0.695(88)$ \\
\hline
\end{tabular}

\begin{tabular}{|c|c|c|c|}
\hline \multirow{2}{*}{ Fluxes } & \multicolumn{3}{|c|}{$\boldsymbol{Y}(\mathbf{m m}), \boldsymbol{Z}=\mathbf{5 2 4} \mathbf{~ m m}$} \\
\cline { 2 - 4 } & $\mathbf{- 4 0}$ & $\mathbf{- 8 0}$ & $\mathbf{- 1 2 0}$ \\
\hline$\varphi_{1 E x p}$ & $424(45)$ & $350(40)$ & $303(32)$ \\
$\varphi_{1 \text { Cal }} / \varphi_{1 \operatorname{Exp}}$ & $0.869(93)$ & $0.89(10)$ & $0.800(95)$ \\
\hline$\varphi_{2 \operatorname{Exp}}$ & $2.02(22)$ & $1.12(13)$ & $0.75(12)$ \\
$\varphi_{2 \text { Cal }} / \varphi_{2 \operatorname{Exp}}$ & $10.4(11)$ & $11.8(14)$ & $10.8(18)$ \\
\hline$\varphi_{3 \operatorname{Exp}}$ & $0.413(42)$ & $0.209(18)$ & $0.110(30)$ \\
$\varphi_{3 \text { Cal }} / \varphi_{3 \operatorname{Exp}}$ & $2.25(23)$ & $2.15(19)$ & $2.10(58)$ \\
\hline$\varphi_{4 E x p}$ & $0.275(29)$ & $0.143(16)$ & $0.105(18)$ \\
$\varphi_{4 \text { Cal }} / \varphi_{4 \operatorname{Exp}}$ & $1.27(14)$ & $1.19(17)$ & $0.84(15)$ \\
\hline$\varphi_{5 \operatorname{Exp}}$ & $0.0505(56)$ & $0.0251(35)$ & $0.0154(27)$ \\
$\varphi_{5 \text { Cal }} / \varphi_{5 \operatorname{Exp}}$ & $0.694(77)$ & $0.686(97)$ & $0.61(10)$ \\
\hline \multicolumn{4}{|c}{}
\end{tabular}

Table 5: Experimental values of the neutron flux in different positions (neutron $\cdot$ deuteron ${ }^{-1} \cdot \mathrm{MeV}^{-1} \cdot \mathrm{cm}^{-2}$. $10^{-3}$ ) and comparison with simulation at the various sections of QUINTA target

\section{Cross-section measurement of residual nuclei}

The supplementary part of the ADS related research at the JINR is a measurement and completion of missing data in nuclear databases, like the EXFOR database [16]. The growing interest in nuclear codes validations in the end of 90's led to a pilot experiments focused on a thin target made of natural uranium irradiation using the direct kinematics method [17]. That means, that in 


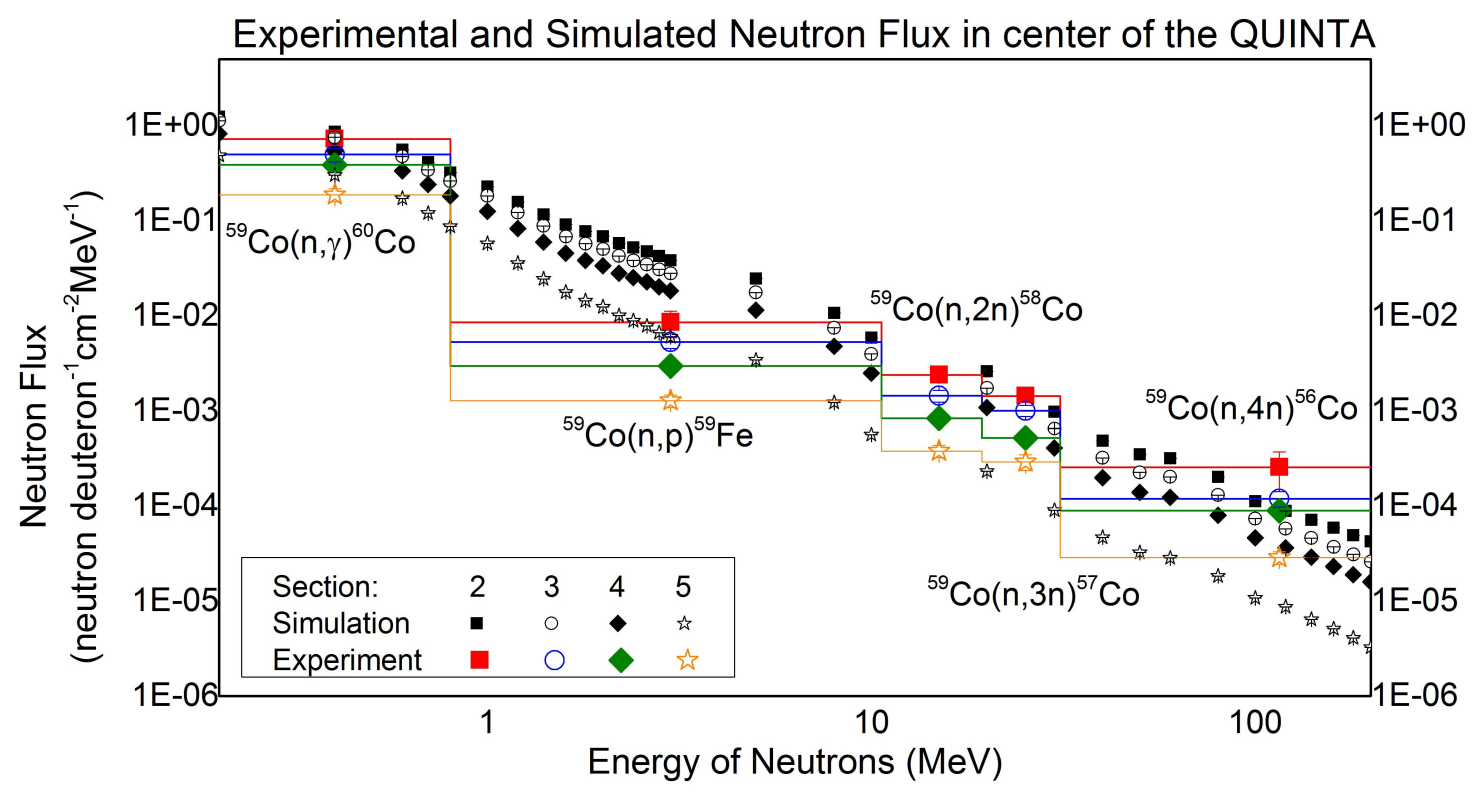

Figure 3: A comparison of experimental and simulated neutron fluxes at the center of the QUINTA target

contrary to the inverse kinematics experiments, thin target made of heavy element was irradiated with a beam of light particles like protons or deuteron.

Most of experiments were performed using the aforementioned Phasotron accelerator. The practical component of this accelerator is a special hydraulic water-cooled holder, which provides an opportunity to irradiate targets within a vacuum chamber with protons of kinetic energy in the range from $60 \mathrm{MeV}$ up to $660 \mathrm{MeV}$. In practice, it is possible to irradiate experimental samples inside the accelerator with much higher intensities in comparison to derived beam.

As a continuation of the study of residual nuclei produced in the natural uranium, in 2014 started irradiations of thin thorium targets inside the Phasotron accelerator with different kinetic energies, namely $200 \mathrm{MeV}$ and $400 \mathrm{MeV}$. Each experiment consisted of two separate irradiations which differs in the irradiation time. Whereas a "short" irradiation time and minimal distance between the accelerator facility and spectroscopic laboratory allowed us to measure short-living isotopes with half-lifes from several minutes, a "long" irradiation created a sufficiently high amount of long-living isotopes allowing long-term measurements. In 2016, another two irradiations were performed with a proton beam of a kinetic energy $660 \mathrm{MeV}$.

Experimental technique was similar to a procedure sketched out in the section 3 with the use of gamma-spectroscopy technique. For cross-section calculation, in-house developed code enabling decay curves fitting was used. Detailed description of calculation procedure can be found in a paper [18].

As stated above, the MCNPX2.7 Monte-Carlo code [12] serves as an important tool for theoretical predictions of aforementioned spallation set-ups. Contrary to the standard reactor applications, there are no evaluated nuclear data libraries in the particles's energy range above $20 \mathrm{MeV}$ (150 Mev in the case of some isotopes). Therefore, MCNPX2.7 incorporates several theoretical models describing high-energy nucleon induced reactions, like spallation, in order to calculate 


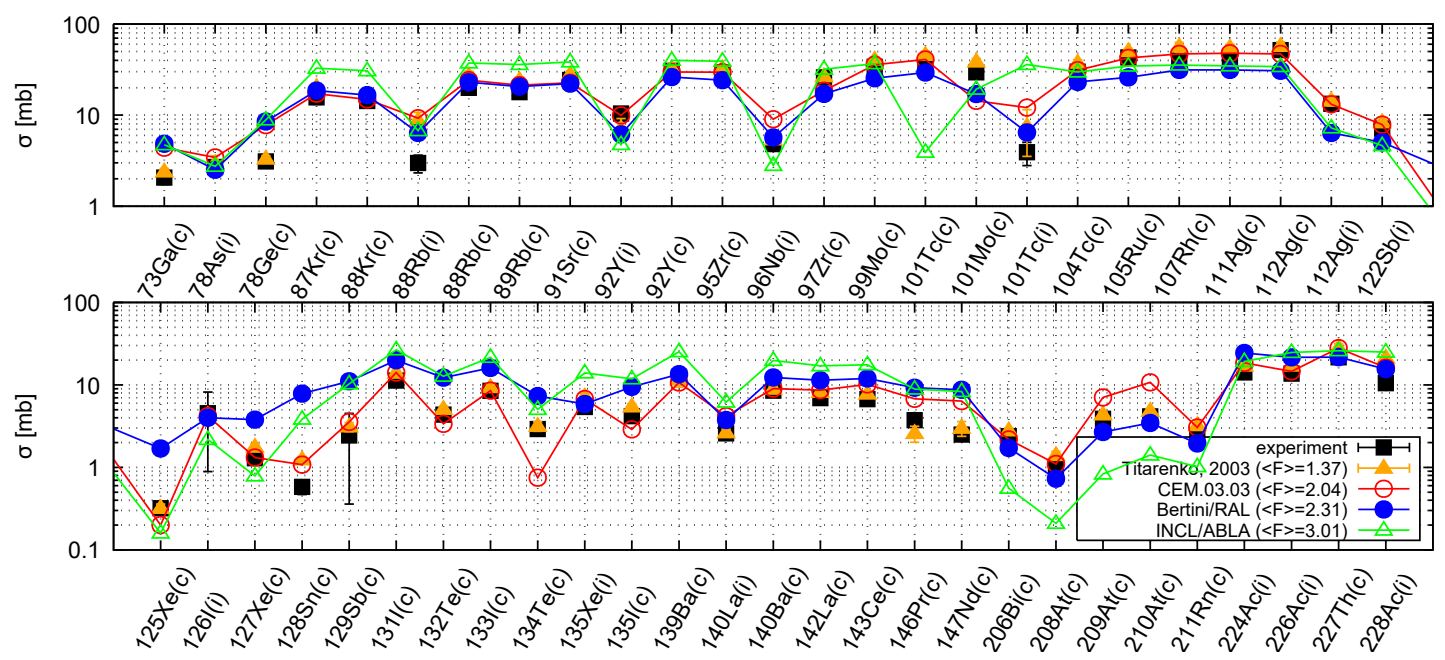

Figure 4: Detailed comparison between selected measured cross-sections from $200 \mathrm{MeV}$ proton irradiation of a thin thorium target ("i" stands for independent and "c" for cumulative) and the predictions of CEM.03.03, Bertini/RAL and INCL4.2/ABLA high-energy event generators implemented in MCNPX2.7 transport code and previously measured results by Titarenko et. al [19].

particle's cross-sections and other necessary quantities. In the low energy range , CEM03.03, INCL4.2, Bertini and ISABEL models are implemented in the MCNPX2.7 code. However, in the higher energy range above approximately $1 \mathrm{GeV}$, there are only two possibilities, the default Los Alamos Quark Gluon String Model (LAQGSM03.03) and the INCL4.2 high-energy event generators. However, MCNPX2.7 transport code is only capable to calculate independent cross-sections, therefore, the cumulative ones were reconstructed using the decay schemes of measured isotopes.

The quantitative comparison between experimental and simulated cross-sections was made by assessing the mean-squared deviation factor $\langle F\rangle$ defined by the equation:

$$
\langle F\rangle=10 \sqrt{\left\langle\left(\log \left(\sigma_{\text {calc }, i}\right)-\log \left(\sigma_{\text {exp }, i}\right)\right)^{2}\right\rangle},
$$

where \langle\rangle stands for averaging over all the experimental and simulated results used in the comparison.

Figure 4 shows an example of comparison between experimental and calculated cross-sections in a case of $200 \mathrm{MeV}$ irradiation of natural thorium. Measured cross-sections were compared with results previously measured by Titarenko et. al. [19] and with theoretical predictions of selected high-energy event generators implemented in the MCNPX2.7 code. Generally, the data are in a reasonably good agreement with an exception of several isotopes, like ${ }^{128} \mathrm{Sn}$. This disagreements will be seriously examined at future experiments with different beam energies.

Similar thin target experiment was also performed at the Nuclotron accelerator. Experimental stack composed of natural uranium, thorium and aluminium monitors was irradiated using a deuteron beam of total kinetic energy 3.5 GeV/A. However, much lower beam intensity and a longer time interval between the end of irradiation and the measurement start (approx. 1 hour) allowed us to measure significantly lower number of residual nuclei (over 80 including independent 
and cumulative ones). Nevertheless, measured data are still valuable, since similar data are missing in the EXFOR database in this energy range.

\section{Conclusion}

The paper summarizes present work of the part of E\&T RAW group working at the DLNP at the JINR which is interested in ADS relared research for more than 20 years. The experimental work developed from relatively small spallation targets up to huge spallation target made from depleted uranium.

Part of the experimental work was focused on the determination of the spallation target QUINTA with the use of threshold activation detectors. The 10 samples of ${ }^{59} \mathrm{Co}$ were irradiated inside of the spallation target. The irradiated samples were measured and mean value of the experimental neutron flux were calculated from experimental reaction rates. The experimental neutron flux was the highest at the position $-40 \mathrm{~mm}$ behind section 2 . The neutron flux was decreasing with increasing longitudinal and vertical distance. The density of the neutron flux in the center of the QUINTA target was approximately decreased of $25 \%$ per section. The highest neutron flux was about $10 \%$ higher than neutron flux in the center behind section 2 . The differences between neutron flux in the center of the spallation target and position $-120 \mathrm{~mm}$ were approximately $30 \%$ for section 2 and 4 .

The final part of the paper deals with residual nuclei cross-sections measurements. Several experiments using the direct kinematics technique with a natural thorium samples are described. Providing new data is especially important for validation of nuclear codes describing various stages of the spallation reaction and future development of these models.

\section{Acknowledgments}

This work was performed on the behalf of Energy and Transmutation collaboration at the JINR. At this place, we would like to thank the personnel of Nuclotron and Phasotron accelerators for their technical support during experiments.

\section{References}

[1] M. Manolopoulou, S. Stoulos, M. Fragopoulou, R. Brandt, W. Westmeier, M. Krivopustov et al., Proton and neutron production from GAMMA-2 spallation source irradiated by relativistic proton beams, Nuclear Instruments and Methods in Physics Research Section A: Accelerators, Spectrometers, Detectors and Associated Equipment 586 (2008) 239 - 245.

[2] J.-S. Wan, T. Schmidt, E.-J. Langrock, P. Vater, R. Brandt, J. Adam et al., Transmutation of 129 I and 237Np using spallation neutrons produced by 1.5, 3.7 and 7.4 GeV protons, Nuclear Instruments and Methods in Physics Research Section A: Accelerators, Spectrometers, Detectors and Associated Equipment 463 (2001) 634 - 652.

[3] J. Adam, C. Bhatia, K. Katovsky, V. Kumar, M. Majerle, V. S. Pronskikh et al., A study of reaction rates of $(n, f),(n, \gamma)$ and $(n, 2 n)$ reactions in nat $U$ and 232 Th by the neutron fluence produced in the graphite set-up (GAMMA-3) irradiated by $2.33 \mathrm{GeV}$ deuteron beam, The European Physical Journal A 47 (2011) 1-18. 
[4] J. Adam, K. Katovsky, M. Majerle, M. I. Krivopustov, V. Kumar, C. Bhatia et al., A study of nuclear transmutation of Th and nat $U$ with neutrons produced in a Pb target and $U$ blanket irradiated by 1.6 GeV deuterons, The European Physical Journal A 43 (2010) 159-173.

[5] J. Borger, S. Hashemi-Nezhad, D. Alexiev, R. Brandt, W. Westmeier, B. Thomauske et al., Studies of the neutron field of the Energy plus Transmutation set-up under $4 \mathrm{GeV}$ deuteron irradiation, Radiation Measurements 46 (2011) 1765 - 1769.

[6] L. Zavorka, J. Adam, M. Artiushenko, A. Baldin, V. Brudanin, O. Bukhal et al., Validation of Monte Carlo simulation of neutron production in a spallation experiment, Annals of Nuclear Energy 80 (2015) 178 - 187.

[7] M. Suchopar, V. Wagner, O. Svoboda, J. Vrzalova, P. Chudoba, A. Kugler et al., Cross-section studies of relativistic deuteron reactions on copper by activation method, Nuclear Instruments and Methods in Physics Research Section B: Beam Interactions with Materials and Atoms 344 (2015) 63 - 69.

[8] J. Khushvaktov, J. Adam, A. Baldin, V. Chilap, V. Furman, F. Sagimbaeva et al., Interactions of secondary particles with thorium samples in the setup \{QUINTA\} irradiated with $6 \mathrm{GeV}$ deuterons, Nuclear Instruments and Methods in Physics Research Section B: Beam Interactions with Materials and Atoms 381 (2016) 84 - 89.

[9] W. Furman, J. Adam, M. Artyushenko, A. Baldin, A. Berlev, M. Bielevicz et al., Recent results of the study of ADS with $500 \mathrm{~kg}$ natural uranium target assembly QUINTA irradiated by deuterons with energies from 1 to $8 \mathrm{GeV}$ at JINR NUCLOTRON , PoS, XXI Baldin ISHEPP, September 10-15, 2012. Dubna, PoS (Baldin ISHEP XXI)086 (2012) .

[10] J. Frána, Program deimos32 for gamma-ray spectra evaluation, Journal of Radioanalytical and Nuclear Chemistry 257 (2003) 583-587.

[11] J. Adam, A. R. Balabekyan, V. S. Barashenkov, R. Brandt, V. M. Golovatiouk, V. G. Kalinnikov et al., Spallation neutron spectrum on a massive lead/paraffin target irradiated with $1 \mathrm{GeV}$ protons, The European Physical Journal A - Hadrons and Nuclei 23 (2005) 61-68.

[12] D. Pelowitz, “MCNPX users manual version 2.7.0 LA-CP-11-00438.” 2011.

[13] A. Boudard, J. Cugnon, S. Leray and C. Volant, Intranuclear cascade model for a comprehensive description of spallation reaction data, Phys. Rev. C 66 (Oct, 2002) 044615.

[14] J. Cugnon, C. Volant and S. Vuillier, Nucleon and deuteron induced spallation reactions, Nuclear Physics A 625 (1997) 729 - 757.

[15] A. Junghans, M. de Jong, H.-G. Clerc, A. Ignatyuk, G. Kudyaev and K.-H. Schmidt, Projectile-fragment yields as a probe for the collective enhancement in the nuclear level density, Nuclear Physics A 629 (1998) 635-655. 
[16] N. Otuka, E. Dupont, V. Semkova, B. Pritychenko, A. Blokhin, M. Aikawa et al., Towards a more complete and accurate experimental nuclear reaction data library (exfor):

International collaboration between nuclear reaction data centres ( $n r d c)$, Nuclear Data Sheets 120 (2014) 272 - 276.

[17] J. Adam, K. Katovsky, R. Michel, A. Balabekyan, V. M. Tsoupko-Sitnikov, V. G. Kalinnikov et al., Investigation of Formation of Residual Nuclei from natU by Reactions with $660 \mathrm{MeV}$ Protons, AIP Conference Proceedings 769 (2005) 1043-1046.

[18] I. Adam, J. Mrazek, J. Frana, A. R. Balabekyan, V. Pronskikh, V. Kalinnikov et al., Software for calculating nuclear reaction cross sections, Measurement Techniques 44 (2001) 93-100.

[19] Y. E. Titarenko, Experimental yields for Th-232 irradiated with 0.1, 0.2, 0.8, 1.2 and 1.6-GeV protons, Tech. Rep. 839B, 1999. 